\title{
On Isoperimetric Profiles of Product Spaces
}

\author{
Thierry Coulhon, Alexander Grigor'yan, ANd Daniel Levin
}

Let $p \in[1,+\infty]$. Given the $L^{p}$-isoperimetric profile of two noncompact Riemannian manifolds $M$ and $N$, we compute the $L^{p_{-}}$ isoperimetric profile of the product $M \times N$.

\section{Introduction.}

We start with an almost tautological identity for the product of Euclidean spaces: $\mathbb{R}^{d}=\mathbb{R}^{n} \times \mathbb{R}^{m}$, where $d=m+n$. This simple fact says that dimensions of Euclidean spaces add up under direct product. The aim of this paper is to show that this fact admits an analogue for isoperimetric dimensions of non-compact Riemannian manifolds (and more general spaces, see Section 7 below). As we will see, the correct notion of dimension here is not a number, but rather a family of functions indexed by a parameter $p \in[1,+\infty]$, the family of $p$-isoperimetric profiles; we shall give a formula that enables one to compute this family of functions for a product, given the ones associated with the factors, and generalizes the addition of dimensions in the Euclidean case.

\section{1. $p$-isoperimetric profiles of non-compact Riemannian mani-} folds. Let $M$ be a Riemannian manifold. The notion of topological dimension of $M$ does not reflect the geometry of $M$ in the large. One of the ways to capture the large scale structure of $M$ is by using isoperimetric inequalities. Let $\mu$ be the Riemanniain measure on $M$, or more generally, a measure with a positive $C^{\infty}$ density $\sigma$ with respect to the Riemannian measure. We shall call $(M, \mu)$ a weighted Riemannian manifold. For any open set $\Omega \subset M$,

\footnotetext{
${ }^{1}$ Research of Thierry Coulhon was partially supported by the European Commission (European TMR Network "Harmonic Analysis" 1998-2001, Contract ERBFMRX-CT97-0159). Research of Daniel Levin was supported by a Chateaubriand Fellowship and the above European TMR Network. Thierry Coulhon and Alexander Grigor'yan were partially supported by the British-French Alliance Programme Project no. 03049SC. Alexander Grigor'yan also acknowledges the support of the University of Cergy-Pontoise.
} 
denote $|\Omega|=\mu(\Omega)$. On any hypersurface $S$ in $M$, consider the surface measure having the density $\sigma$ with respect to the Riemannian surface measure; let $|S|$ be the full surface measure of $S$. Denote by $\operatorname{Lip}_{0}(M)$ the space of Lipschitz functions with compact support on $M$.

If, for some $d>1, C>0$ and for any non-empty precompact open set $\Omega \subset M$ with smooth boundary,

$$
|\Omega|^{\frac{d-1}{d}} \leq C|\partial \Omega|,
$$

then it is natural to say that $M$ has isoperimetric dimension $d$. It is known ([36], [53]) that (1.1) is equivalent to the following Sobolev inequality: for any function $f \in \operatorname{Lip}_{0}(M)$

$$
\left(\int_{M}|f|^{\frac{d}{d-1}} d \mu\right)^{\frac{d-1}{d}} \leq C \int_{M}|\nabla f| d \mu .
$$

Alongside $\left(S_{d}^{1}\right)$, one can consider a more general family of Sobolev inequalities of the form

$$
\left(\int_{M}|f|^{\frac{p d}{d-p}} d \mu\right)^{\frac{d-p}{d}} \leq C_{p} \int_{M}|\nabla f|^{p} d \mu
$$

for any $f \in \operatorname{Lip}_{0}(M)$, assuming $1 \leq p<d$. If $\left(S_{d}^{p}\right)$ holds, then one says that $M$ has $p$-isoperimetric dimension $d$. The idea that such Sobolev inequalities carry some large scale dimensional information on Riemannian manifolds can be traced back at least to [61] and [54, Sect. 3].

One can also write down a natural version of $\left(S_{d}^{p}\right)$ for $d<p<+\infty$ (the so-called Gagliardo-Nirenberg inequalities), for $p=d$ (the Trudinger-Moser inequality) and even for $p=+\infty$ (see [24]). Then one observes that $\left(S_{d}^{\infty}\right)$ is equivalent to the volume lower bound

$$
V(x, r) \geq c r^{d}, \quad \forall x \in M, \forall r>0,
$$

where $V(x, r)$ is the volume $\mu(B(x, r))$ of the geodesic ball $B(x, r)$ on $M$ of radius $r$ centered at $x$. It is possible to show that $\left(S_{d}^{p}\right) \Longrightarrow\left(S_{d}^{q}\right)$ for all $1 \leq p<q \leq+\infty$ (see [15], [24]) but the converse is in general false (see [29], [14], [4]).

The importance of Sobolev inequalities for analysis on manifolds is well known (see for instance [1], [17], [44], [47], [48], [49], [50], [58]). For example, Varopoulos [61] proved that, for $d>2,\left(S_{d}^{2}\right)$ is equivalent to the heat kernel upper estimate

$$
\sup _{x \in M} p_{t}(x, x) \leq C t^{-d / 2}, \quad \forall t>0,
$$


where $p_{t}(x, y)(t>0, x, y \in M)$ is the heat kernel of $(M, \mu)$, that is the minimal positive fundamental solution to the heat equation $\partial_{t} u=\Delta u$ on $M \times \mathbb{R}_{+}$. Here $\Delta$ is the Laplace operator of the weighted manifold $(M, \mu)$, that is the generator associated with the Dirichlet form $f \mapsto \int|\nabla f|^{2} d \mu$ in $L^{2}(M, \mu)$ with domain $\operatorname{Lip}_{0}(M)$ or $C_{0}^{\infty}(M)$.

However, the nice picture above is spoiled by the fact that even very simple manifolds may not have any isoperimetric dimension. For example, none of the inequalities $\left(S_{d}^{p}\right)$ takes place for the cylinder $M=\mathbb{R}^{m} \times \mathbb{S}^{n-m}$, $n>m$. Indeed, $\left(S_{d}^{p}\right)$ holds for functions $f$ with small supports if and only if $n \leq d$ since locally $M$ looks like $\mathbb{R}^{n}$. On the other hand, if $\left(S_{d}^{p}\right)$ is true then it implies $\left(S_{d}^{\infty}\right)$, that is $V(x, r) \geq c r^{d}$, whereas for large $r$ we have $V(x, r) \leq$ $\mathrm{Cr}^{m}$. Hence, we obtain $n \leq d \leq m$ which contradicts the assumption $n>m$. The point of this argument is that the local topological dimension of a manifold and its asymptotic dimension at infinity may be different, in which case the Sobolev inequality $\left(S_{d}^{p}\right)$ cannot be satisfied.

A way to overcome this difficulty consists in localizing properly the Sobolev inequalities (see [18], [21], [22], [31]) so that one distinguishes the local dimension and the dimension at infinity. This approach is satisfactory, say for polynomial volume growth Lie groups (see for instance, [65]), but already for Lie groups with exponential volume growth, Sobolev inequalities are not well adapted (cf. [64]).

On the other hand, it is easy to generalize the isoperimetric inequality (1.1) so that it would take place on a much larger class of manifolds including those mentioned above. Given a non-negative non-increasing function $\psi$ on ] $0,+\infty[$, consider instead of (1.1) the following isoperimetric inequality

$$
\psi(|\Omega|)|\Omega| \leq|\partial \Omega| .
$$

Clearly, (1.1) is a particular case of (1.2) for $\psi(v)=c v^{-1 / d}$. The cylinder $\mathbb{R}^{m} \times \mathbb{S}^{n-m}$ admits (1.2) with the function

$$
\psi(v)= \begin{cases}c_{1} v^{-1 / n}, & v \leq 1 \\ c_{2} v^{-1 / m}, & v>1\end{cases}
$$

Lie groups with polynomial growth lead to an isoperimetric profile of a similar form. Examples of a different kind are as follows. On unimodular amenable Lie groups with exponential volume growth, one obtains

$$
\psi(v)= \begin{cases}c_{1} v^{-1 / n}, & v \leq 2 \\ \frac{c_{2}}{\log (v)}, & v>2\end{cases}
$$


and on co-compact covering manifolds

$$
\psi(v)= \begin{cases}c_{1} v^{-1 / n}, & v \leq 1 \\ \frac{c_{2}}{V^{-1}(C v)}, & v>1\end{cases}
$$

where $V$ is the volume growth function of the deck transformation group (see [30]). More examples of isoperimetric inequalities on manifolds and their applications can be found in [16], [17], [19], [41], [42], [51], [68], [69].

Following [23], let us introduce a general $(p, \psi)$-isoperimetric inequality on $M$ as follows: for any precompact open set $\Omega \subset M$ and for any function $f \in \operatorname{Lip}_{0}(\Omega)$,

$$
\psi(|\Omega|)\|f\|_{p} \leq\||\nabla f|\|_{p} .
$$

Here $\psi$ is a non-negative non-increasing function on $(0,+\infty), p \in[1,+\infty]$, and $\|\cdot\|_{p}$ is the $L^{p}(M, \mu)$-norm.

It is possible to show that if $\psi(v)=c v^{-1 / d}$ (we shall refer to this as the polynomial case) then $\left(S_{\psi}^{p}\right)$ is equivalent to $\left(S_{d}^{p}\right)$, which justifies our notation (see [15], [24], [3]). Again, $\left(S_{\psi}^{1}\right)$ is equivalent to $(1.2)$, and $\left(S_{\psi}^{\infty}\right)$ is equivalent to

$$
V(x, r) \geq \psi^{-1}(1 / r), \forall x \in M, r>0 .
$$

Also, for $1 \leq p<q<+\infty,\left(S_{\psi}^{p}\right)$ implies $\left(S_{\psi}^{q}\right)$. However, contrary to the polynomial case, it is no more true in general that $\left(S_{\psi}^{p}\right)$ with $1 \leq p<+\infty$ implies $\left(S_{\psi}^{\infty}\right)$ (this follows from [56]).

Denote by $\lambda_{1}(\Omega)$ the bottom of the spectrum of $-\Delta$ in $L^{2}(\Omega, \mu)$, that is

$$
\lambda_{1}(\Omega)=\inf _{\substack{f \in \operatorname{Lip}_{0}(\Omega) \\ f \neq 0}} \frac{\int|\nabla f|^{2} d \mu}{\int f^{2} d \mu} .
$$

Then $\left(S_{\psi}^{2}\right)$ can be rewritten as the Faber-Krahn inequality

$$
\lambda_{1}(\Omega) \geq \psi^{2}(|\Omega|)
$$

for any non-empty precompact open set $\Omega \subset M$. This inequality was introduced in [39], [40] to investigate various aspects of the heat kernel behavior. In particular, it was proved in [40] that under certain regularity assumptions about $\psi,(1.3)$ is equivalent (up to constant multiples) to the heat kernel estimate

$$
\sup _{x \in M} p_{t}(x, x) \leq \frac{1}{\varphi(t)}, \quad \forall t>0
$$


where the functions $\varphi$ and $\psi$ are related by

$$
\frac{\varphi^{\prime}(t)}{\varphi(t)}=\psi^{2}(\varphi(t)), \quad \varphi(0)=0
$$

- see also Section 6 below. Another application of $\left(S_{\psi}^{p}\right)$ is related to the notion of $p$-hyperbolicity (see [28, Sect. 3.3], [38], [40]). For details see Section 5 below.

Given a weighted Riemannian manifold $M$, with each $p \in[1,+\infty]$ one can associate the largest function $\psi$ such that $\left(S_{\psi}^{p}\right)$ holds on $M$ :

$$
\psi_{M, p}(v)=\inf \left\{\frac{\|\nabla f \mid\|_{p}}{\|f\|_{p}}: f \in \operatorname{Lip}_{0}(\Omega) \backslash\{0\},|\Omega|=v\right\},
$$

where $\Omega$ is a precompact open subset of $M$, and the infimum is taken over all $f$ and $\Omega$ as specified. The function $\psi_{M, p}$ is automatically non-increasing (but it can vanish). We shall call it the $p$-isoperimetric profile. This notion was introduced in [26], see also [28, Sect. 3.3], although there one considers $\varphi=\frac{1}{\psi}$ rather than $\psi$. In the examples considered above, the $p$-isoperimetric profile does not depend on $p$ : up to multiplicative constants $\psi_{M, p}=\psi$, $p \in[1,+\infty]$; this fact contains a lot of non-trivial information, concerning for instance the connection between the heat kernel decay and the volume growth (see [27]). But this connection is not as tight in general (see [4]), and, as we already mentioned in the polynomial case, the $p$-isoperimetric profile does depend on $p$; one can show that if $1 \leq p \leq q<+\infty$, then

$$
\psi_{M, q}(v) \geq c(p, q) \psi_{M, p}(v)
$$

but conversely (if $M$ has bounded geometry) one only has

$$
\psi_{M, q}^{q}(v) \leq C\left(p, q, v_{0}\right) \psi_{M, p}(v), \quad v \geq v_{0},
$$

and the examples in [29], [14] show that this is sharp.

1.2. The cases $p=1,2,+\infty$. Now we can come back to our initial question on the isoperimetric dimension of product manifolds, and reformulate it in the following way: given two weighted Riemannian manifolds $(M, \mu)$ and $(N, \nu)$ that satisfy respectively $\left(S_{\psi_{M}}^{p}\right)$ and $\left(S_{\psi_{N}}^{p}\right)$ for some $p \in[1,+\infty]$, which inequality $\left(S_{\psi}^{p}\right)$ does the product $(M \times N, \mu \times \nu)$ satisfy?

Before we start, let us recall that the question of the behaviour of Sobolev and isoperimetric inequalities under products has recently received a lot of attention in other contexts (see for instance [11], [12], [47], p.330, and [70], $\S 2)$. 
In the polynomial case, if the Riemannian manifolds $M$ and $N$ satisfy respectively $\left(S_{m}^{p}\right)$ and $\left(S_{n}^{p}\right)$ for $1 \leq p<\min (m, n)$, then the Riemannian product $M \times N$ satisfies $\left(S_{n+m}^{p}\right)$. However, this is not so easy to prove. This was done by Varopoulos (see [62], [63]), using the interpolation inequality for mixed norms [9], see also in [18] the remarks after Prop. 4; a more detailed proof can be found in [59] (in the setting of graphs).

Outside the polynomial setting, the only case which was investigated so far is $p=1$. The case $p=+\infty$ is easy, and the case $p=2$ can be settled by a heat kernel argument. Let us briefly outline the results in these cases, assuming in each case that $M$ and $N$ satisfy respectively the $\left(p, \psi_{M}\right)$ - and $\left(p, \psi_{N}\right)$-isoperimetric inequalities.

Case $p=1$. It was proved by one of the authors [37, Theorem 2] that the product $M \times N$ satisfies the $\left(1, \frac{1}{3} \psi\right)$-isoperimetric inequality with

$$
\psi(w):=\inf _{u v=w}\left(\psi_{M}(u)+\psi_{N}(v)\right),
$$

assuming that the functions $\psi_{M}(u) u$ and $\psi_{N}(v) v$ are increasing. Note that the techniques of [37] are very specific to the case $p=1$.

The shape of (1.7) is very natural from the point of view of (1.2). Indeed, consider the particular case when the set $\Omega$ is a product $\Omega_{M} \times \Omega_{N}$, where $\Omega_{M}$ and $\Omega_{N}$ are precompact open sets with smooth boundaries in $M$ and $N$ respectively. Then $\partial \Omega$ is the union of $\partial \Omega_{M} \times \Omega_{N}$ and $\Omega_{M} \times \partial \Omega_{N}$, whence

$$
|\partial \Omega|=\left|\partial \Omega_{M}\right|\left|\Omega_{N}\right|+\left|\Omega_{M}\right|\left|\partial \Omega_{N}\right| .
$$

Since $|\Omega|=\left|\Omega_{M}\right|\left|\Omega_{N}\right|$, we obtain

$$
\frac{|\partial \Omega|}{|\Omega|}=\frac{\left|\partial \Omega_{M}\right|}{\left|\Omega_{M}\right|}+\frac{\left|\partial \Omega_{N}\right|}{\left|\Omega_{N}\right|} \geq \psi_{M}\left(\left|\Omega_{M}\right|\right)+\psi_{N}\left(\left|\Omega_{N}\right|\right) \geq \psi(|\Omega|) .
$$

On the other hand, if the $\left(1, \psi_{M}\right)$ - and $\left(1, \psi_{N}\right)$-isoperimetric inequalities on $M$ and $N$ are sharp, this argument shows that one cannot get better than $(1, \psi)$-isoperimetric inequality on $M \times N$. In other terms, if $\psi_{M}, \psi_{N}$, and $\psi_{M \times N}$ are the 1-isoperimetric profiles respectively of $M, N$, and $M \times N$, then

$$
\frac{1}{3} \psi \leq \psi_{M \times N} \leq \psi
$$

where $\psi$ is defined by (1.7).

Case $p=2$. This case can also be treated by a specific method, using the aforementioned equivalence of the $(2, \psi)$-isoperimetric inequality and the 
heat kernel decay (1.4) (in the next argument, we skip the constant factors). Indeed, the heat kernels on $M$ and $N$ admit the estimates

$$
\sup _{x \in M} p_{t}^{(M)}(x, x) \leq \frac{1}{\varphi_{M}(t)} \quad \text { and } \quad \sup _{y \in N} p_{t}^{(N)}(y, y) \leq \frac{1}{\varphi_{N}(t)}
$$

where $\varphi_{M}$ and $\varphi_{N}$ are determined by (1.5). The heat kernel $p_{t}$ on $M \times N$ is the product of $p_{t}^{(M)}$ and $p_{t}^{(N)}$ in the following sense:

$$
\text { if } z=(x, y) \in M \times N \text { then } p_{t}(z, z)=p_{t}^{(M)}(x, x) p_{t}^{(N)}(y, y)
$$

(see for example, [42, Section 1.5]). Hence, we obtain

$$
\sup _{z \in M \times N} p_{t}(z, z) \leq \frac{1}{\varphi_{M}(t) \varphi_{N}(t)}=\frac{1}{\varphi(t)}
$$

where $\varphi=\varphi_{M} \varphi_{\widetilde{N}}$. Therefore, $M \times N$ satisfies the $(2, \widetilde{\psi})$-isoperimetric inequality, where $\widetilde{\psi}$ is determined by

$$
\widetilde{\psi}^{2}(\varphi)=\frac{\varphi^{\prime}}{\varphi}=\frac{\varphi_{M}^{\prime}}{\varphi_{M}}+\frac{\varphi_{N}^{\prime}}{\varphi_{N}}=\psi_{M}^{2}\left(\varphi_{M}\right)+\psi_{N}^{2}\left(\varphi_{N}\right) .
$$

Consequently, if we define $\psi$ by

$$
\psi^{2}(w):=\inf _{u v=w}\left(\psi_{M}^{2}(u)+\psi_{N}^{2}(v)\right),
$$

then $\widetilde{\psi} \geq \psi$ so that the $(2, \psi)$-isoperimetric inequality holds on $M \times N$.

Case $p=+\infty$. For any $z=(x, y) \in M \times N$, the geodesic ball $B(z, \sqrt{2} r)$ in $M \times N$ contains $B_{M}(x, r) \times B_{N}(y, r)$, whence

$$
V(z, \sqrt{2} r) \geq V_{M}(x, r) V_{N}(y, r) \geq \psi_{M}^{-1}(1 / r) \psi_{N}^{-1}(1 / r) .
$$

Defining the function $\psi$ by

$$
\psi^{-1}(s)=\psi_{M}^{-1}(s) \psi_{N}^{-1}(s),
$$

we see that $M \times N$ satisfies the $(\infty, \widetilde{\psi})$-isoperimetric inequality, where $\widetilde{\psi}(w)=\psi(\sqrt{2} w)$. Setting in (1.9) $u_{0}=\psi_{M}^{-1}(s)$ and $v_{0}=\psi_{N}^{-1}(s)$, we obtain

$$
\psi\left(u_{0} v_{0}\right)=\psi_{M}\left(u_{0}\right)=\psi_{N}\left(v_{0}\right),
$$

which implies that $\psi$ can also be defined by

$$
\psi(w)=\inf _{u v=w} \max \left(\psi_{M}(u), \psi_{N}(v)\right) .
$$

1.3. Statement of the main result. We are now in a position to formulate the following generalization of (1.7), (1.8), (1.10) which is the main result of this paper. 
Theorem 1.1. Let $p \in[1,+\infty]$. Suppose that the weighted Riemannian manifolds $(M, \mu)$ and $(N, \nu)$ satisfy respectively the $\left(p, \psi_{M}\right)$ - and $\left(p, \psi_{N}\right)$ isoperimetric inequalities, where $\psi_{M}$ and $\psi_{N}$ are non-negative and nonincreasing on $(0,+\infty)$. Define the function $\psi$ by

$$
\psi(w):=\inf _{u v=w}\left(\psi_{M}^{p}(u)+\psi_{N}^{p}(v)\right)^{1 / p}
$$

for $p<+\infty$, with the obvious modification, i.e. (1.10), for $p=+\infty$. Then, for any $\theta>1$, the product manifold $(M \times N, \mu \times \nu)$ satisfies the $(p, \widetilde{\psi})$ isoperimetric inequality where

$$
\widetilde{\psi}(w):=c \psi(\theta w)
$$

and $c=c(p, \theta)>0$.

If in addition for some $\alpha>0$ one of the functions $\psi_{M}(u) u^{\alpha}$ and $\psi_{N}(v) v^{\alpha}$ is increasing, then $\theta$ can be taken 1 .

Of course, since finally we allow a multiplicative constant in front of $\psi$, we could suppress $p$ in formula (1.11) and write instead

$$
\psi(w):=\inf _{u v=w}\left(\psi_{M}(u)+\psi_{N}(v)\right) .
$$

But the advantage of formula (1.11) is that it admits (1.10) as a limit case for $p=+\infty$.

The fact that formula (1.12) is the same for every $p \in[1,+\infty[$ implies that the operation of taking finite products is not able to create substantial differences between $p$-isoperimetric profiles, contrary to the constructions in [29], [14] or [4].

It is an easy exercise to check that, if $\psi_{M}(u)=c_{1} u^{-1 / n}$ and $\psi_{N}(v)=$ $c_{2} v^{-1 / m}$, then formula (1.12) yields $\psi(w)=c w^{-1 / d}$, with $d=m+n$. A more exotic family of examples is treated in Section 5 below.

An important point is that (1.12) is sharp: if $\psi_{M}, \psi_{N}$, and $\psi_{M \times N}$ are the $p$-isoperimetric profiles respectively of $M, N$, and $M \times N$, then

$$
c \psi(\theta w) \leq \psi_{M \times N}(w) \leq \psi(w),
$$

for any $\theta>1$ and $c=c(p, \theta)>0$. Indeed, for all $u, v>0$ and any $\varepsilon>0$ there exist precompact open sets with smooth boundaries $\Omega_{M}$ and $\Omega_{N}$ in $M$ and $N$ respectively and $f \in \operatorname{Lip}_{0}\left(\Omega_{M}\right) \backslash\{0\}, g \in \operatorname{Lip}_{0}\left(\Omega_{N}\right) \backslash\{0\}$, such that

$$
\left|\Omega_{M}\right|=u, \quad\left|\Omega_{N}\right|=v
$$


and

$$
\||\nabla f|\|_{p} \leq(1+\varepsilon) \psi_{M}\left(\left|\Omega_{M}\right|\right)\|f\|_{p}, \quad\|\nabla g \mid\|_{p} \leq(1+\varepsilon) \psi_{N}\left(\left|\Omega_{N}\right|\right)\|g\|_{p} .
$$

Now let $w>0$, and let $u, v$ be such that $u v=w$ and

$$
\psi_{M}(u)+\psi_{N}(v) \leq(1+\varepsilon) \psi(w) .
$$

Consider $\Omega_{M}, \Omega_{N}, f, g$, associated with $u$ and $v$ as above, and set

$$
h=f g \in \operatorname{Lip}_{0}\left(\Omega_{M} \times \Omega_{M}\right) .
$$

Then

$$
\|h\|_{p}=\|f\|_{p}\|g\|_{p},
$$

and, since $\nabla h=g \nabla f+f \nabla g$,

$$
\||\nabla h|\|_{p} \leq\|g\|_{p}\||\nabla f|\|_{p}+\|f\|_{p}\||\nabla g|\|_{p} .
$$

It follows that

$$
\begin{aligned}
\frac{\||\nabla h|\|_{p}}{\|h\|_{p}} \leq \frac{\||\nabla f|\|_{p}}{\|f\|_{p}} & +\frac{\|\nabla g \mid\|_{p}}{\|g\|_{p}} \leq(1+\varepsilon)\left(\psi_{M}\left(\left|\Omega_{M}\right|\right)+\psi_{N}\left(\left|\Omega_{N}\right|\right)\right) \\
& \leq(1+\varepsilon)^{2} \psi\left(\left|\Omega_{M} \times \Omega_{N}\right|\right) .
\end{aligned}
$$

Finally, since $\varepsilon>0$ is arbitrary, we obtain the upper bound in (1.13). Of course, the lower bound follows from Theorem 1.1.

Our method of proof of Theorem 1.1 is based on the observation that the $(p, \psi)$-isoperimetric inequality is equivalent to another kind of functional inequality which we call F-Sobolev inequality, following F-Y. Wang ([66]) who considered it in the case $p=2$. This inequality appeared also in [20] and [10], also for $p=2$.

Let $F$ be a non-decreasing non-negative function on $[0,+\infty[$ and let $p \in$ $\left[1,+\infty\left[\right.\right.$. We say that $M$ satisfies the $F$-Sobolev inequality in $L^{p}$ if, for any $f \in \operatorname{Lip}_{0}(M), f \not \equiv 0$,

$$
\int|f|^{p} F\left(\frac{|f|^{p}}{\|f\|_{p}^{p}}\right) d \mu \leq \int|\nabla f|^{p} d \mu .
$$

Our second main observation is that it is relatively simple to deduce such an inequality on a product manifold from similar inequalities on the factors.

A famous example of a $F$-Sobolev inequality is the so-called $L^{2}$ Moser inequality

$$
A_{n} \int_{\mathbb{R}^{n}}|f(x)|^{2+\frac{4}{n}} d x \leq\left(\int_{\mathbb{R}^{n}}|f(x)|^{2} d x\right)^{2 / n} \int_{\mathbb{R}^{n}}|\nabla f(x)|^{2} d x,
$$


which holds in $\mathbb{R}^{n}$; here $p=2$ and $F(r)=A_{n} r^{2 / n}$. In the $L^{p}$ version of this inequality, one would find $F(r)=A_{n, p} r^{p / n}$. This example shows that contrary to $\psi$ in $(p, \psi)$-isoperimetric inequalities, $F$ contains generically a dependence on $p$; in this respect, the correct object to consider would be $F^{1 / p}$ rather than $F$. This will appear in the relationship we shall establish below between $F$ and $\psi$.

In the polynomial case, our point can therefore be summarized in the following way: on the one hand, Moser inequalities are easily seen to be "multipliable", and on the other hand they are equivalent to the more familiar Sobolev inequalities (this already follows from [3]). This yields an alternative proof of the Varopoulos result on products of Sobolev inequalities mentioned above.

In Section 2 we prove, following Wang, that $F$-Sobolev inequalities are equivalent to suitable $(p, \psi)$-isoperimetric inequalities. In Section 3 , we prove that $F$-Sobolev inequalities are multipliable. Theorem 1.1 is proved in Section 4. In Section 5 we treat a family of examples and show an application of Theorem 1.1 to the $p$-hyperbolicity of a product manifold, where one is led to estimate the $p$-isoperimetric profile, for $p \neq 1,2,+\infty$, in a non-polynomial situation. In Section 6, we examine the relationship between $F$-Sobolev inequalities, $(p, \psi)$-isoperimetric inequalities, and one-parameter log-Sobolev inequalities. In Section 7, we put our results into the general framework of [3]. Finally, in an appendix, we use Proposition 3.1 (the fact that $F$-Sobolev inequalities are multipliable) to give a new proof of the Sobolev inequalities in the Euclidean space, and to prove the Moser inequality (1.14) with $A_{n}=\frac{\pi^{2}}{4} n$, which gives the correct rate of growth of the sharp constant as $n \rightarrow \infty$. A similar result is proved in the case $1 \leq p \leq 2$.

\section{Equivalence of $\psi$-isoperimetric and $F$-Sobolev inequalities.}

In this section we show that, for $p \in[1,+\infty[$, the $(p, \psi)$-isoperimetric inequality is equivalent to a certain $F$-Sobolev inequality, up to constant multiples. Our approach is similar to the one in [66, Thms 3.1 and 3.2] and [10], although these works treated only the case $p=2$. One of the differences with our approach is that we consider $(p, \psi)$-isoperimetric inequalities, whereas [66] works with a so-called $\beta$-Nash inequality, and [10] works with a generalized Nash inequality (introduced in [60] and [25]). It is possible to show that a $\beta$-Nash inequality can be reduced to a generalized Nash inequality by optimizing on the parameter, and a generalized Nash inequality is equivalent to a $(p, \psi)$-isoperimetric inequality (see [3, Prop.10.3]). Hence, in some sense 
our approach is equivalent to that in [66] and [10], although technically our proofs are simpler, avoiding difficulties related to inversion of a Legendre transform (cf. also Section 6).

As in [66], [10], the path from $(p, \psi)$-isoperimetric inequalities to $F$ Sobolev uses the truncation technique already exploited in [3], whereas the converse implication is more direct.

Proposition 2.1. Let $p \in[1,+\infty[$ and assume that $(M, \mu)$ satisfies the $(p, \psi)$-isoperimetric inequality. Then, for any $\eta>1,(M, \mu)$ satisfies the $F$-Sobolev inequality in $L^{p}$ with

$$
F(r):=c \psi^{p}\left(\frac{\eta}{r}\right)
$$

and $c=c(p, \eta)>0$.

Proof: Let $f \in \operatorname{Lip}_{0}(M)$ and suppose that

$$
\int_{M}|f|^{p} d \mu=1
$$

Fix $\rho>1$ and define, for any $k \in \mathbb{Z}$, the set

$$
\Omega_{k}=\left\{x \in M:|f(x)| \geq \rho^{k}\right\}
$$

and the function $f_{k}$ by

$$
f_{k}=\min \left(\left(|f|-\rho^{k}\right)_{+},\left(\rho^{k+1}-\rho^{k}\right)\right) .
$$

One has

$$
\left|\Omega_{k}\right|=\mu\left\{|f| \geq \rho^{k}\right\} \leq \rho^{-p k} \int|f|^{p} d \mu=\rho^{-p k} .
$$

Applying the $(p, \psi)$-isoperimetric inequality to each $f_{k}$ and observing that $f_{k} \in \operatorname{Lip}_{0}\left(\Omega_{k}\right)$, we obtain

$$
\int\left|\nabla f_{k}\right|^{p} d \mu \geq \psi^{p}\left(\left|\Omega_{k}\right|\right) \int f_{k}^{p} d \mu \geq \psi^{p}\left(\rho^{-p k}\right) \int f_{k}^{p} d \mu,
$$

since $\psi$ is non-increasing. It is also clear that

$$
\int f_{k}^{p} d \mu \geq \int_{\Omega_{k+1}} f_{k}^{p} d \mu=\left|\Omega_{k+1}\right|\left(\rho^{k+1}-\rho^{k}\right)^{p} .
$$

Combining with

$$
\int_{M}|\nabla f|^{p} d \mu \geq \sum_{k \in \mathbb{Z}} \int_{\Omega_{k} \backslash \Omega_{k+1}}|\nabla f|^{p} d \mu=\sum_{k \in \mathbb{Z}} \int\left|\nabla f_{k}\right|^{p} d \mu
$$


and denoting

$$
a:=\frac{(\rho-1)^{p}}{1-\rho^{-p}}=\frac{\left(\rho^{k+1}-\rho^{k}\right)^{p}}{\rho^{p k}-\rho^{p(k-1)}}
$$

we obtain

$$
\begin{aligned}
\int_{M}|\nabla f|^{p} d \mu & \geq \sum_{k \in \mathbb{Z}} \psi^{p}\left(\rho^{-p k}\right)\left|\Omega_{k+1}\right|\left(\rho^{k+1}-\rho^{k}\right)^{p} \\
& =a \sum_{k \in \mathbb{Z}} \psi^{p}\left(\rho^{-p k}\right) \mu\left\{|f|^{p} \geq \rho^{p(k+1)}\right\}\left(\rho^{p k}-\rho^{p(k-1)}\right) \\
& \geq a \sum_{k \in \mathbb{Z}} \int_{\rho^{p(k-1)}}^{\rho^{p k}} \psi^{p}\left(\frac{1}{t}\right) \mu\left\{|f|^{p} \geq \rho^{2 p} t\right\} d t \\
& =a \int_{0}^{+\infty} \psi^{p}\left(\frac{1}{t}\right) \mu\left\{|f|^{p} \geq \rho^{2 p} t\right\} d t \\
& =b \int_{0}^{+\infty} \psi^{p}\left(\frac{\rho^{2 p}}{s}\right) \mu\left\{|f|^{p} \geq s\right\} d s,
\end{aligned}
$$

where

$$
b:=a \rho^{-2 p}=\frac{(\rho-1)^{p}}{\rho^{2 p}-\rho^{p}}
$$

Define the function $\widetilde{F}$ by

$$
\widetilde{F}(r)=\frac{b}{r} \int_{0}^{r} \psi^{p}\left(\frac{\rho^{2 p}}{s}\right) d s .
$$

Then we have

$$
\begin{aligned}
\int_{M}|f|^{p} \widetilde{F}\left(|f|^{p}\right) d \mu & =b \int_{M}\left(\int_{0}^{|f(x)|^{p}} \psi^{p}\left(\frac{\rho^{2 p}}{s}\right) d s\right) d \mu(x) \\
& =b \int_{0}^{+\infty}\left(\int_{\left\{|f|^{p} \geq s\right\}} d \mu\right) \psi^{p}\left(\frac{\rho^{2 p}}{s}\right) d s \\
& =b \int_{0}^{+\infty} \psi^{p}\left(\frac{\rho^{2 p}}{s}\right) \mu\left\{|f|^{p} \geq s\right\} d s
\end{aligned}
$$

Comparing with (2.2), we see that $M$ satisfies the $\widetilde{F}$-Sobolev inequality.

Let us observe that, for any $0<\varepsilon<1$,

$$
\widetilde{F}(r) \geq \frac{b}{r} \int_{\varepsilon r}^{r} \psi^{p}\left(\frac{\rho^{2 p}}{s}\right) d s \geq b(1-\varepsilon) \psi^{p}\left(\frac{\rho^{2 p}}{\varepsilon r}\right) .
$$


If $1<\rho^{2 p}<\eta$, then take $\varepsilon=\frac{\rho^{2 p}}{\eta}$, which yields

$$
\widetilde{F}(r) \geq b\left(1-\frac{\rho^{2 p}}{\eta}\right) \psi^{p}\left(\frac{\eta}{r}\right) .
$$

Finally, optimizing in $\rho$, we obtain $\widetilde{F}(r) \geq F(r)$ where $F$ is defined by (2.1) and

$$
c=c(p, \eta):=\sup _{1<\rho<\eta^{1 / 2 p}} \frac{(\rho-1)^{p}}{\rho^{2 p}-\rho^{p}}\left(1-\frac{\rho^{2 p}}{\eta}\right) .
$$

Proposition 2.2. Fix $p \in[1,+\infty[$, and assume that $M$ satisfies the $F$ Sobolev inequality in $L^{p}$. Then for any $0<\varepsilon<1, M$ satisfies the $(p, \psi)$ isoperimetric inequality with $\psi$ defined by

$$
\psi^{p}(w)=(1-\varepsilon) F\left(\frac{\varepsilon}{w}\right) .
$$

Proof: Let $\Omega$ be a precompact open subset of $M$ and let $f \in \operatorname{Lip}_{0}(\Omega)$ be a function such that

$$
\int|f|^{p} d \mu=1
$$

Let $F^{-1}$ be the generalized inverse to $F$ defined by

$$
F^{-1}(t)=\inf \{s: F(s) \geq t\} .
$$

Observe that the following inequality is true for all non-negative $s$ and $t$ :

$$
t s \leq s F(s)+t F^{-1}(t) .
$$

Taking here $s=|f|^{p}$ we obtain

$$
t|f|^{p}-t F^{-1}(t) \leq|f|^{p} F\left(|f|^{p}\right) .
$$

Integrating (2.5) over $\Omega$ yields

$$
t \int|f|^{p} d \mu-t F^{-1}(t)|\Omega| \leq \int|f|^{p} F\left(|f|^{p}\right) d \mu .
$$

Applying (2.4) and the F-Sobolev inequality, we obtain

$$
t-t F^{-1}(t)|\Omega| \leq \int|\nabla f|^{p} d \mu .
$$


This inequality is valid for all $t \geq 0$. Fix $\varepsilon \in] 0,1\left[\right.$ and choose $t=F\left(\frac{\varepsilon}{|\Omega|}\right)$ so that $F^{-1}(t) \leq \frac{\varepsilon}{|\Omega|}$. Hence, we obtain

$$
(1-\varepsilon) F\left(\frac{\varepsilon}{|\Omega|}\right) \leq \int|\nabla f|^{p} d \mu
$$

which exactly means that the $(p, \psi)$-isoperimetric inequality is valid with $\psi$ defined by (2.3).

\section{F-Sobolev inequality on a product manifold.}

In this section we shall prove the following.

Proposition 3.1. Let $p \in[1,+\infty[$. Suppose that the weighted Riemannian manifolds $(M, \mu)$ and $(N, \nu)$ satisfy respectively the $F$ - and $G$-Sobolev inequalities in $L^{p}$. Then the product manifold $(M \times N, \mu \times \nu)$ satisfies the $c_{p} H$-Sobolev inequality in $L^{p}$, where the function $H$ is given by the formula

$$
H(r)=\inf _{s t=r}[F(s)+G(t)]
$$

and

$$
c_{p}= \begin{cases}1, & p \geq 2 \\ 2^{(p / 2)-1}, & p<2\end{cases}
$$

Proof: Let $f \in \operatorname{Lip}_{0}(M \times N)$. Without loss of generality, we can assume

$$
\int_{M \times N}|f|^{p} d \mu d \nu=1
$$

Introduce the function

$$
h(y):=\left(\int_{M}|f(x, y)|^{p} d \mu(x)\right)^{\frac{1}{p}} .
$$

Applying the $F$-Sobolev inequality to $f(x, y)$ as a function of $x$ and then integrating in $y$, we obtain

$$
\int_{N} \int_{M}|f|^{p} F\left(\frac{|f|^{p}}{h^{p}}\right) d \mu d \nu \leq \int_{N} \int_{M}\left|\nabla_{x} f\right|^{p} d \mu d \nu .
$$

By (3.2), we have $\|h\|_{p}=1$. Applying the $G$-Sobolev inequality to $h(y)$ (note that $h$ is a Lipschitz function as one can see from (3.6) below) we obtain

$$
\int_{N} \int_{M}|f|^{p} G\left(h^{p}\right) d \mu d \nu=\int_{N} h^{p} G\left(h^{p}\right) d \nu \leq \int_{N}\left|\nabla_{y} h\right|^{p} d \nu .
$$


Let us observe that

$$
\int_{N}\left|\nabla_{y} h\right|^{p} d \nu \leq \int_{M} \int_{N}\left|\nabla_{y} f\right|^{p} d \mu d \nu
$$

Indeed, we have

$$
\begin{aligned}
\nabla_{y} h & =\frac{1}{p}\left(\int_{M} \nabla_{y}|f|^{p} d \mu\right)\left(\int_{M}|f|^{p} d \mu\right)^{\frac{1}{p}-1} \\
& =\left(\int_{M}\left(\nabla_{y}|f|\right)|f|^{p-1} d \mu\right)\left(\int_{M}|f|^{p} d \mu\right)^{\frac{1}{p}-1}
\end{aligned}
$$

whence, by the Hölder inequality,

$$
\left|\nabla_{y} h\right| \leq\left(\int_{M}\left|\nabla_{y} f\right||f|^{p-1} d \mu\right)\left(\int_{M}|f|^{p} d \mu\right)^{\frac{1}{p}-1} \leq\left(\int_{M}\left|\nabla_{y} f\right|^{p} d \mu\right)^{1 / p}
$$

and (3.5) follows.

Summing up (3.3), (3.4) and using (3.5), we obtain

$$
\begin{gathered}
\int_{N} \int_{M}|f|^{p} F\left(\frac{|f|^{p}}{h^{p}}\right) d \mu d \nu+\int_{N} \int_{M}|f|^{p} G\left(h^{p}\right) d \mu d \nu \\
\leq \int_{N} \int_{M}\left|\nabla_{x} f\right|^{p} d \mu d \nu+\int_{N} \int_{M}\left|\nabla_{y} f\right|^{p} d \mu d \nu .
\end{gathered}
$$

Now, by the definition of the product metric on $M \times N$, we have

$$
|\nabla f|^{2}=\left|\nabla_{x} f\right|^{2}+\left|\nabla_{y} f\right|^{2}
$$

Therefore,

$$
|\nabla f|^{p}=\left(\left|\nabla_{x} f\right|^{2}+\left|\nabla_{y} f\right|^{2}\right)^{p / 2} \geq c_{p}\left(\left|\nabla_{x} f\right|^{p}+\left|\nabla_{y} f\right|^{p}\right)
$$

where $c_{p}$ is defined by (3.1). Thus

$\int_{N} \int_{M}|f|^{p} F\left(\frac{|f|^{p}}{h^{p}}\right) d \mu d \nu+\int_{N} \int_{M}|f|^{p} G\left(h^{p}\right) d \mu d \nu \leq c_{p}^{-1} \int_{N} \int_{M}|\nabla f|^{p} d \mu d \nu$.

In order to estimate the left-hand side of (3.7) from below, we use the definition of function $H$ which implies

$$
F\left(\frac{|f|^{p}}{h^{p}}\right)+G\left(h^{p}\right) \geq H\left(|f|^{p}\right)
$$


Therefore (3.7) yields

$$
\int_{N} \int_{M}|f|^{p} H\left(|f|^{p}\right) d \mu d \nu \leq c_{p}^{-1} \int_{N} \int_{M}|\nabla f|^{p} d \mu d \nu
$$

which is exactly the $c_{p} H$-Sobolev inequality on $M \times N$.

\section{Proof of the main theorem.}

We are finally in a position to prove Theorem 1.1. The case $p=+\infty$ has already been treated, let therefore $p \in[1,+\infty[$. Assuming that the manifolds $M$ and $N$ satisfy respectively the $\left(p, \psi_{M}\right)$ - and $\left(p, \psi_{N}\right)$-isoperimetric inequalities, Proposition 2.1 says that they also satisfy the $c F_{M^{-}}$and $c F_{N}$-Sobolev inequalities in $L^{p}$, where

$$
F_{M}(r)=c \psi_{M}^{p}\left(\frac{\eta}{r}\right) \quad \text { and } \quad F_{N}(r)=c \psi_{N}^{p}\left(\frac{\eta}{r}\right),
$$

$\eta>1$ is arbitrary and $c=c(p, \eta)>0$. By Proposition $3.1, M \times N$ satisfies the $F$-Sobolev inequality where $F$ is defined by

$$
\begin{aligned}
F(r) & =c_{p} \inf _{s t=r}\left[F_{M}(s)+F_{N}(t)\right] \\
& =c c_{p} \inf _{s t=r}\left[\psi_{M}^{p}\left(\frac{\eta}{s}\right)+\psi_{N}^{p}\left(\frac{\eta}{t}\right)\right] \\
& =c c_{p} \inf _{u v=\eta^{2} / r}\left[\psi_{M}^{p}(u)+\psi_{N}^{p}(v)\right] \\
& =c c_{p} \psi^{p}\left(\frac{\eta^{2}}{r}\right),
\end{aligned}
$$

the function $\psi$ being defined by (1.11). By Proposition 2.2, $M \times N$ satisfies the $(p, \widetilde{\psi})$-isoperimetric inequality where

$$
\widetilde{\psi}^{p}(w):=(1-\varepsilon) F\left(\frac{\varepsilon}{w}\right)=(1-\varepsilon) c c_{p} \psi^{p}\left(\varepsilon^{-1} \eta^{2} w\right) .
$$

We are left to observe that $\theta:=\varepsilon^{-1} \eta^{2}>1$ can be made arbitrarily close to one.

If, say, $\psi_{M}(u) u^{\alpha}$ is increasing for some $\alpha>0$, then we see from

$$
\left(\psi(w) w^{\alpha}\right)^{p}=\inf _{v>0}\left[\left(\psi_{M}\left(\frac{w}{v}\right)\left(\frac{w}{v}\right)^{\alpha}\right)^{p} v^{\alpha p}+\psi_{N}^{p}(v) w^{\alpha p}\right]
$$

that $\psi(w) w^{\alpha}$ is non-decreasing. Therefore, for any $\theta>1$, we have $\psi(\theta w) \theta^{\alpha} w^{\alpha} \geq \psi(w) w^{\alpha}$ and $\psi(\theta w) \geq \theta^{-\alpha} \psi(w)$, which settles the second claim of Theorem 1.1. 


\section{An example and an application.}

To motivate our family of examples, suppose that the heat kernel on a manifold $M$ admits the following upper bound

$$
\sup _{x \in M} p_{t}^{(M)}(x, x) \leq \frac{C}{t^{\alpha} \log ^{\gamma} t}, \quad t \geq t_{0}>0,
$$

with $\alpha>0$ and $\gamma \in \mathbb{R}$ (such examples can be found in [4]). By using [40, Theorem 2.2], (5.1) implies that $M$ satisfies the $(2, \psi)$-isoperimetric inequality, with

$$
\psi^{2}(v)=c v^{-\frac{1}{\alpha}}(\log v)^{\frac{\gamma}{\alpha}}, \quad v \geq v_{0}>0 .
$$

Let now $p \in[1,+\infty[$. Suppose that $M$ and $N$ satisfy respectively the $\left(p, \psi_{M}\right)$ - and $\left(p, \psi_{N}\right)$-isoperimetric inequalities, with $\psi_{M}$ and $\psi_{N}$ such that

$$
\psi_{M}^{p}(u)=c_{1} u^{-\frac{1}{\alpha}}(\log u)^{\delta_{1}}, \quad u \geq u_{0}>1,
$$

and

$$
\psi_{N}^{p}(v)=c_{2} v^{-\frac{1}{\beta}}(\log v)^{\delta_{2}}, \quad v \geq v_{0}>1,
$$

where $\alpha, \beta>0$ and $\delta_{1}, \delta_{2} \in \mathbb{R}$. Using Theorem 1.1, one can check that $M \times N$ satisfies the $\left(p, \psi_{M \times N}\right)$-isoperimetric inequality with

$$
\psi_{M \times N}^{p}(w)=c w^{-\frac{1}{\alpha+\beta}}(\log w)^{\delta_{1} \frac{\alpha}{\alpha+\beta}+\delta_{2} \frac{\beta}{\alpha+\beta}}, \quad w \geq w_{0},
$$

for a large enough $w_{0}$ and some $c>0$. In order to estimate

$$
\inf _{u v=w}\left[\psi_{M}^{p}(u)+\psi_{N}^{p}(v)\right]
$$

let us first observe that

$$
\inf _{u v=w, u \leq u_{0}}\left[\psi_{M}^{p}(u)+\psi_{N}^{p}(v)\right] \geq \inf _{u \leq u_{0}} \psi_{M}^{p}(u) \geq \psi_{M}^{p}\left(u_{0}\right),
$$

since $\psi_{M}$ is non-increasing. Similarly,

$$
\inf _{u v=w, v \leq v_{0}}\left[\psi_{M}^{p}(u)+\psi_{N}^{p}(v)\right] \geq \psi_{N}^{p}\left(v_{0}\right) .
$$

Finally, to estimate

$$
\inf _{u v=w, u \geq u_{0}, v \geq v_{0}}\left[\psi_{M}^{p}(u)+\psi_{N}^{p}(v)\right],
$$


observe that the infimum in (5.4) is attained when the two summands are comparable, which is the case for

$$
u=w^{\frac{\alpha}{\alpha+\beta}}(\log w)^{\delta_{3}},
$$

where $\delta_{3}=\left(\delta_{1}-\delta_{2}\right)\left(\frac{1}{\alpha}+\frac{1}{\beta}\right)^{-1}$. Note that if $w$ is large enough then $u>u_{0}$ and $v:=w / u>v_{0}$. Substituting (5.5) into (5.4) and taking into account that the infima in (5.2) and (5.3) are bounded by positive constants, we obtain the claim.

In [28, Theorem 3.3], it was shown that if $1<p<\infty$ and $M$ satisfies the $(p, \psi)$-isoperimetric inequality with a function $\psi$ such that

$$
\int^{+\infty} \frac{d v}{(v \psi(v))^{\frac{p}{p-1}}}<+\infty
$$

then $M$ is $p$-hyperbolic (the case $p=2$ was treated before in [40, Theorem $2.3]$; see also [43, Section 10]). For example, if $\psi(v)=c v^{-\frac{1}{p}} \log ^{\theta} v$, for large $v$, then $M$ is $p$-hyperbolic provided $\theta>\frac{p-1}{p}$.

Suppose that $(M, \mu)$ satisfies the $\left(p, \psi_{1}\right)$-isoperimetric inequality with $\psi_{1}(u)=c_{1} u^{-\frac{2}{p}} \log ^{\theta_{1}} u$, for large $u$, and that $(N, \nu)$ satisfies the $\left(p, \psi_{2}\right)$ isoperimetric inequality with $\psi_{2}(v)=c_{2} v^{-\frac{2}{p}} \log ^{\theta_{2}} v$, for large $v$, where $\theta_{1}$ and $\theta_{2}$ are real numbers. We have just seen that the $(p, \psi)$-isoperimetric inequality on $(M \times N, \mu \times \nu)$ holds with the function

$$
\psi(w)=c w^{-\frac{1}{p}} \log ^{\left(\theta_{1}+\theta_{2}\right) / 2} w
$$

for large $w$. Hence, we conclude that $(M \times N, \mu \times \nu)$ is $p$-hyperbolic provided $\theta_{1}+\theta_{2}>\frac{2(p-1)}{p}$.

\section{ultracontractivity.}

\section{One-parameter log-Sobolev inequalities and}

Given $1 \leq p<+\infty$ and a decreasing ${ }^{1}$ function $m(t):(0,+\infty) \rightarrow \mathbb{R}$, we say that the $m$-log-Sobolev inequality holds in $L^{p}(M, \mu)$ if

$$
\int_{M}|f|^{p} \log \left(\frac{|f|^{p}}{\|f\|_{p}^{p}}\right) d \mu \leq t \int_{M}|\nabla f|^{p} d \mu+m(t) \int_{M}|f|^{p} d \mu
$$

\footnotetext{
${ }^{1}$ We understand the terms "decreasing" and "increasing" in the non-strict sense, that is, as synonyms for "non-increasing" and "non-decreasing", respectively.
} 
for all $f \in \operatorname{Lip}_{0}(M), f \not \equiv 0$, and all $t>0$. Such inequalities were introduced by Davies and Simon [35] and were intensively used to investigate the decay of the heat semigroup (see for example [32], [33]). If one optimises in $t$ in (6.1), one obtains a form of inequality which was introduced for $p=2$ by Bakry under the name energy-entropy inequality (see [1], [2]).

Let $\Delta$ be the Laplace operator of $(M, \mu)$. We say that the heat semigroup $\left\{e^{t \Delta}\right\}_{t \geq 0}$ is m-ultracontractive if for all $t>0$

$$
\left\|e^{t \Delta}\right\|_{1 \rightarrow \infty} \leq e^{m(t)}
$$

By [34, Theorem 2.2.3], if $e^{t \Delta}$ is $m$-ultracontractive then the $m$-log-Sobolev inequality holds, with the same function $m$. Conversely, by [34, Corollary 2.2.8] if the $m$-log-Sobolev inequality holds in $L^{2}$ then $e^{t \Delta}$ is $\widetilde{m}$ ultracontractive where

$$
\widetilde{m}(t)=\frac{1}{t} \int_{0}^{t} m(\tau) d \tau
$$

provided $\widetilde{m}(t)$ is finite.

It is well known in various contexts that log-Sobolev inequalities behave nicely with respect to taking direct product of the underlying spaces (see for instance [5, p.108]). This is also the case for (6.1) as is shown in the following statement (the proof is similar to the one of Proposition 3.1 and is omitted).

Proposition 6.1. If the $m_{1}$ - and $m_{2}$-log-Sobolev inequalities hold in $L^{p}(M, \mu)$ and $L^{p}(N, \nu)$ respectively, then the $m$-log-Sobolev inequality holds in $L^{p}(M \times N, \mu \times \nu)$, where $m=c_{p}\left(m_{1}+m_{2}\right)$ and $c_{p}$ is the constant defined by (3.1).

In this section, we will establish a direct link between the $F$-Sobolev and the $m$-log-Sobolev inequalities. Together with Proposition 6.1 , this yields an alternative route for computing the $(p, \psi)$-isoperimetric inequalities on product manifolds, although this route is longer than the one used in the proof of Theorem 1.1.

Theorem 6.2. Let $1 \leq p<+\infty$.

(a) If the $F$-Sobolev inequality holds in $L^{p}(M, \mu)$ then the $m$-log-Sobolev inequality holds in $L^{p}(M, \mu)$ where

$$
m(t):=\sup _{s>0}\{\log s-t F(s)\}
$$

provided the right-hand side of (6.3) is finite. 
(b) If the $m$-log-Sobolev inequality holds in $L^{p}(M, \mu)$ then the $\widetilde{F}$-Sobolev inequality holds in $L^{p}(M, \mu)$ with function

$$
\widetilde{F}(r)=c F(r / \eta)
$$

for any $\eta>1$ and $c=c(p, \eta)>0$, where

$$
F(s):=\sup _{t>0} \frac{1}{t}\{\log s-m(t)\}
$$

provided the right-hand side of (6.5) is finite and non-negative.

Remark 6.3. It is clear that the function $m$ obtained by (6.3) is decreasing, and the function $F$ obtained by (6.5) is increasing.

Remark 6.4. In the case $p=2$, a very close result was proved by Biroli and Maheux [10]. Namely, they showed the equivalence of the $F$-Sobolev inequality and the $m$-log-Sobolev inequality in $L^{2}$ via two intermediate steps - a generalized Nash inequality and an energy-entropy inequality. Part $(a)$ for $p=2$ was also observed in [57].

Proof: $(a)$ Let $f \in \operatorname{Lip}_{0}(M)$ and $\|f\|_{p}=1$. By the definition (6.3) of $m(t)$, we have for all $t, s>0$

$$
\log s \leq t F(s)+m(t) .
$$

Multiplying this inequality by $s$, setting $s=|f|^{p}$, and integrating over $M$ we obtain

$$
\int|f|^{p} \log |f|^{p} d \mu \leq t \int|f|^{p} F\left(|f|^{p}\right) d \mu+m(t) \int|f|^{p} d \mu .
$$

Applying the $F$-Sobolev inequality yields (6.1).

(b) As an intermediate step, we first prove that the $m$-log-Sobolev inequality in $L^{p}(M, \mu)$ implies the $(p, \psi)$-isoperimetric inequality where $\psi$ is defined by

$$
\psi^{p}(v):=\sup _{t>0} \frac{1}{t}\left\{\log \frac{1}{v}-m(t)\right\} .
$$

Consider first the case $p>1$. Let $\Omega$ be a non-empty precompact open subset of $M$, and let $f \in \operatorname{Lip}_{0}(\Omega),\|f\|_{p}=1$. We start with Jensen's inequality:

$$
\begin{gathered}
-\log \int|f| d \mu=-\log \int|f|^{1-p}|f|^{p} d \mu \\
\leq-\int\left(\log |f|^{1-p}\right)|f|^{p} d \mu=\frac{p-1}{p} \int|f|^{p} \log |f|^{p} d \mu .
\end{gathered}
$$


Hence, the $m$-log-Sobolev inequality (6.1) implies

$$
-\frac{p}{p-1} \log \int|f| d \mu \leq t \int|\nabla f|^{p} d \mu+m(t)
$$

The Hölder inequality and $\|f\|_{p}=1$ yield

$$
\int_{\Omega}|f| d \mu \leq|\Omega|^{\frac{p-1}{p}}\left(\int|f|^{p} d \mu\right)^{\frac{1}{p}}=|\Omega|^{\frac{p-1}{p}} .
$$

Therefore, combining (6.7) and (6.8), we obtain

$$
-\log |\Omega| \leq t \int|\nabla f|^{p} d \mu+m(t)
$$

and hence

$$
\frac{1}{t}\left(\log \frac{1}{|\Omega|}-m(t)\right) \leq \int|\nabla f|^{p} d \mu .
$$

Taking sup in $t$ yields

$$
\psi^{p}(|\Omega|) \leq \int|\nabla f|^{p} d \mu,
$$

that is the $(p, \psi)$-isoperimetric inequality.

In the case $p=1$ we argue slightly differently. Assuming that $f \in$ $\operatorname{Lip}_{0}(\Omega),\|f\|_{1}=1$, and using Jensen's inequality, we have

$$
\begin{gathered}
-\log \int|f|^{1 / 2} d \mu=-\log \int|f|^{-1 / 2}|f| d \mu \\
\leq-\int\left(\log |f|^{-1 / 2}\right)|f| d \mu=\frac{1}{2} \int|f| \log |f| d \mu .
\end{gathered}
$$

Then (6.1) implies

$$
-2 \log \int|f|^{1 / 2} d \mu \leq t \int|\nabla f| d \mu+m(t) .
$$

On the other hand, by the Cauchy-Schwarz inequality,

$$
\int_{\Omega}|f|^{1 / 2} d \mu \leq|\Omega|^{1 / 2}\left(\int|f| d \mu\right)^{1 / 2}=|\Omega|^{1 / 2},
$$

whence

$$
-\log |\Omega| \leq t \int|\nabla f| d \mu+m(t)
$$


One finishes the proof as above in the case $p>1$.

By Proposition 2.1, the $(p, \psi)$-isoperimetric inequality implies the $\widetilde{F}$ Sobolev inequality in $L^{p}$ with the function $\widetilde{F}(s)=c F(s / \eta)$ where $F(s)=$ $\psi^{p}(1 / s)$. Combining this result with $(6.6)$, we conclude the proof.

We summarize the above proof of Theorem 6.2 in the following diagram:
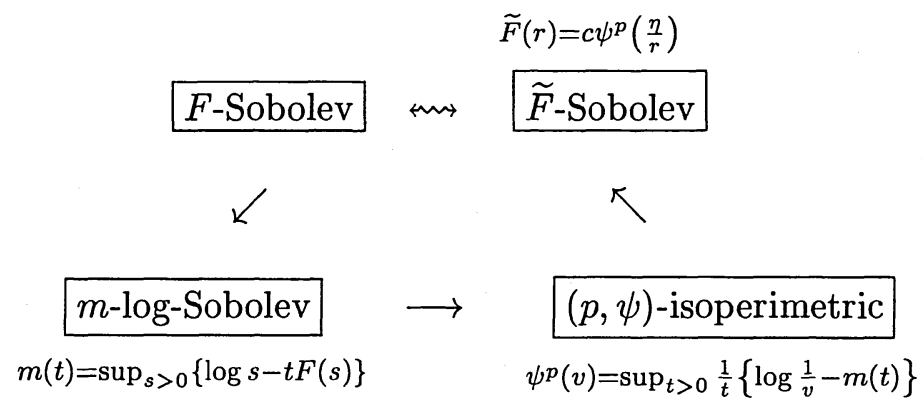

Let us introduce the functions

$$
f(\sigma)=F\left(e^{\sigma}\right) \quad \text { and } \quad g(\tau)=\tau m(1 / \tau)
$$

Then the equations (6.5) and (6.3) can be rewritten as follows:

$$
f(\sigma)=\sup _{\tau \in \mathbb{R}_{+}}\{\tau \sigma-g(\tau)\} \quad \text { and } \quad g(\tau)=\sup _{\sigma \in \mathbb{R}}\{\tau \sigma-f(\sigma)\} .
$$

In other words, the functions $f$ and $g$ are related by the Legendre transform.

Consider the following two functional classes

$$
\mathcal{C}_{+}=\left\{f: \mathbb{R} \rightarrow \mathbb{R}_{+} \mid f \text { is convex, increasing, } \lim _{\sigma \rightarrow+\infty} \frac{f(\sigma)}{\sigma}=+\infty\right\}
$$

and

$$
\mathcal{C}=\left\{g: \mathbb{R}_{+} \rightarrow \mathbb{R} \mid g \text { is convex, } g(0) \leq 0, \lim _{\tau \rightarrow+\infty} \frac{g(\tau)}{\tau}=+\infty\right\}
$$

(where $\mathbb{R}_{+}=[0,+\infty)$ ). The condition $g(0) \leq 0$ in $(6.13)$ can be replaced by the requirement that $g(\tau) / \tau$ is increasing.

Denote by $\mathcal{L}$ the Legendre transform on the class $\mathcal{C}$, and by $\mathcal{L}_{+}$the Legendre transform on the class $\mathcal{C}_{+}$, given by (6.11). An elementary argument shows that $\mathcal{L}$ maps $\mathcal{C}$ to $\mathcal{C}_{+}$and $\mathcal{L}_{+}$maps $\mathcal{C}_{+}$to $\mathcal{C}$; moreover, $\mathcal{L}$ and $\mathcal{L}_{+}$are mutually inverse. We skip this argument but indicate the following points:

- the condition $\lim _{\sigma \rightarrow+\infty} f(\sigma) / \sigma=+\infty$ ensures the finiteness of $g$ (the same applies to the finiteness of $f$ ); 
- the condition $g(0) \leq 0$ is equivalent to the non-negativity of $f$;

- the fact that $\mathcal{L}$ and $\mathcal{L}_{+}$are the mutually inverse transforms roots in the observation that the derivatives $f^{\prime}$ and $g^{\prime}$ are mutually inverse functions.

Consider also the following functional classes:

$\mathcal{F}=\left\{F: \mathbb{R}_{+} \rightarrow \mathbb{R}_{+} \mid F(s)\right.$ and $s F^{\prime}(s)$ are increasing, $\left.\lim _{s \rightarrow+\infty} \frac{F(s)}{\log s}=+\infty\right\}$,

assuming that $F$ is absolutely continuous so that $F^{\prime}$ makes sense, and

$$
\mathcal{M}=\left\{m: \mathbb{R}_{+}^{*} \rightarrow \mathbb{R} \mid m \text { is convex, decreasing, } m(0+)=+\infty\right\} .
$$

It is easy to show that

$$
F(s) \in \mathcal{F} \Longleftrightarrow F\left(e^{\sigma}\right) \in \mathcal{C}_{+} \quad \text { and } \quad m(t) \in \mathcal{M} \Longleftrightarrow \tau m(1 / \tau) \in \mathcal{C} .
$$

Consequently, the relations (6.3), (6.5) provide a bijection between $\mathcal{F}$ and $\mathcal{M}$, and we obtain the following statement.

Corollary 6.5. If $F \in \mathcal{F}$, then starting with the $F$-Sobolev inequality and making a loop in the diagram (6.9), we arrive at the $\widetilde{F}$-Sobolev inequality with $\widetilde{F}(s)=c F(s / \eta)$.

Alternatively, one can say that if $m \in \mathcal{M}$ then a loop on the diagram (6.9) returns to the initial hypothesis, up to constant multiples.

Note that the condition $\lim _{s \rightarrow+\infty} F(s) / \log s=+\infty$ in the definition of $\mathcal{F}$ is important. Indeed, if $F(s)=\log s$ (for large $s$ ) then the $F$-Sobolev inequality amounts to a non-parametric log-Sobolev inequality, which is known to be weaker than any $m$-log-Sobolev inequality.

In the case $p=2$ the diagram (6.9) can be complemented by the above mentioned relations between log-Sobolev inequalities and the ultracontractivity of the heat semigroup (see [57, Section 4] for a direct relation between $F$-Sobolev inequalities and ultracontractivity). Consider for comparison another line of implications based on an alternative method of obtaining the ultracontractive estimate (6.2) using a generalized Nash inequality (this method was employed in [40] and [25]).

Given a decreasing non-negative function $\psi$ on $(0,+\infty)$, we say that the $\psi$-Nash inequality holds in $L^{2}(M, \mu)$ if, for any $f \in \operatorname{Lip}_{0}(M), f \not \equiv 0$,

$$
\psi\left(\frac{\|f\|_{1}^{2}}{\|f\|_{2}^{2}}\right)\|f\|_{2} \leq\|\nabla f\|_{2}
$$


It is known (see [3, Theorem 10.3], [40, Lemma 2.1]) that the $(2, \psi)$ isoperimetric inequality implies the $\widetilde{\psi}$-Nash inequality with

$$
\widetilde{\psi}(v)=\frac{1}{2} \psi(4 v) .
$$

Conversely, the $\psi$-Nash inequality implies the $(2, \psi)$-isoperimetric inequality, just by the Cauchy-Schwarz inequality.

By the standard Nash method one deduces from the $\psi$-Nash inequality that $e^{t \Delta}$ is $m$-ultracontractive where $m(t)$ is determined by the differential equation

$$
-\frac{d m}{d t}=\psi^{2}\left(e^{-m}\right), \quad m(0+)=+\infty,
$$

provided such $m$ exists (see for example, [25, Proposition 2.1], [40, Theorem 2.1], [60]). It is easy to see that any $m$ satisfying (6.18) must be in $\mathcal{M}$. On the other hand, if $m \in \mathcal{M}_{0} \subset \mathcal{M}$ where

$\mathcal{M}_{0}=\left\{m \in C^{1}\left(\mathbb{R}_{+}^{*}\right) \mid m\right.$ is convex, $\left.m^{\prime}<0, m(0+)=+\infty, m(+\infty)=-\infty\right\}$, then indeed $m$ solves (6.18); the corresponding class of functions

$$
F(s)=\psi^{2}(1 / s)
$$

is

$$
\mathcal{F}_{0}=\left\{F \in C\left(\mathbb{R}_{+}^{*}\right) \mid F>0, F \text { is increasing, } \int^{+\infty} \frac{d s}{s F(s)}<+\infty\right\}
$$

(cf. [40, Section 2]). There are examples of $\psi$-Nash inequalities which do not imply any ultracontractivity; this follows from [35, Section 6 , Remark 1 , p. 359].

It was observed respectively in [40, Proof of Theorem 2.2] and [25, Proposition II.2] that the $m$-ultracontractivity implies the $(2, \psi)$-isoperimetric and the $\psi$-Nash inequality with

$$
\psi^{2}(v)=\sup _{t>0} \frac{1}{t}\left(\log \frac{1}{v}-m(t)\right),
$$

assuming $m \in \mathcal{M}$. It is worth mentioning that the function $m(t)=$ $\log \left\|e^{t \Delta}\right\|_{1 \rightarrow \infty}$ is always in $\mathcal{M}$. Let us also emphasize a remarkable fact that (6.19) is identical to (6.6) with $p=2$; that is the $m$-log-Sobolev inequality and the $m$-ultracontractivity imply the same $(2, \psi)$-isoperimetric inequality. 
Combining together the above statements and neglecting the constant multiples in (6.17), we obtain one more diagram:

$$
\begin{aligned}
& \widetilde{F}(s)=\sup _{t>0} \frac{1}{t}(\log s-m(t)) \\
& (2, \psi) \text {-isoperimetric } \leadsto \quad(2, \widetilde{\psi}) \text {-isoperimetric } \\
& \begin{array}{cc}
\swarrow & \nwarrow^{m \in \mathcal{M}} \\
\psi \text {-Nash inequality } \stackrel{m \in \mathcal{M}_{0}}{\longrightarrow} \underset{-m^{\prime}=F\left(e^{m}\right), m(0+)=+\infty}{m \text {-ultracontractivity }}
\end{array}
\end{aligned}
$$

Here we use the notation $F(s) \equiv \psi^{2}(1 / s)$ and $\widetilde{F}(s) \equiv \widetilde{\psi}^{2}(1 / s)$.

Let us verify that always $\widetilde{F} \leq F$, as one should expect. Indeed, it suffices to show that for all $\sigma \in \mathbb{R}$ and $t>0$

$$
\frac{1}{t}(\sigma-m(t)) \leq F\left(e^{\sigma}\right) .
$$

If $m(t) \geq \sigma$ then there is nothing to prove. Otherwise, there exists $0<t_{*}<t$ so that $m\left(t_{*}\right)=\sigma$. Using the convexity of $m$ and the intermediate value theorem, we obtain

$$
\frac{1}{t}(\sigma-m(t))=\frac{m\left(t_{*}\right)-m(t)}{t} \leq \frac{m\left(t_{*}\right)-m(t)}{t-t_{*}} \leq-m^{\prime}\left(t_{*}\right)=F\left(e^{\sigma}\right) .
$$

For example, if $m(t)=\exp \left(\frac{1}{t^{\alpha}}\right), \alpha>0$, then for $s$ large enough

$$
F(s)=\alpha \log s(\log \log s)^{\frac{\alpha+1}{\alpha}}
$$

whereas

$$
\widetilde{F}(s) \asymp \log s(\log \log s)^{\frac{1}{\alpha}}<<F(s) .
$$

Situations with such function $m(t)$ were considered in [35], [46], [20], [7], [2], [8].

For any $\delta>0$, introduce the following subclass of $\mathcal{M}_{0}$ :

$$
\mathcal{M}_{\delta}=\left\{m \in \mathcal{M}_{0}:\left|m^{\prime}(2 t)\right| \geq \delta\left|m^{\prime}(t)\right|, \quad \text { for all } t>0\right\} .
$$

We claim that if $m \in \mathcal{M}_{\delta}$ then

$$
\widetilde{F}(s) \geq \frac{\delta}{2} F(s), \quad \text { for all } s>0
$$


(cf. [40]). Indeed, choosing $t$ so that $m(t)=\log s$, we obtain

$$
\begin{gathered}
\widetilde{F}(s) \geq \frac{1}{2 t}(\log s-m(2 t))=\frac{m(t)-m(2 t)}{2 t} \geq-\frac{1}{2} m^{\prime}(2 t) \\
\geq-\frac{\delta}{2} m^{\prime}(t)=\frac{\delta}{2} F\left(e^{m(t)}\right)=\frac{\delta}{2} F(s) .
\end{gathered}
$$

Hence, if $m \in \mathcal{M}_{\delta}$ then the loop on the diagram (6.20) comes back to the initial hypothesis, up to constant multiples.

\section{A more general setting.}

For the sake of exposition, we have so far presented our results in the setting of Riemannian manifolds. They are however valid in the more general setting of [3], which covers other situations such as manifolds endowed with a secondorder subelliptic operator or graphs endowed with a Markov kernel. We explain now the way one should state the above results so that they fit in this setting. We refer to [3, sections 2 and 7] for details on the examples that can be treated in this way.

Fix $1 \leq p<+\infty$. Let $(M, \mu)$ and $(N, \nu)$ be two measured spaces, $\mathcal{F}_{M}$ and $\mathcal{F}_{N}$ some classes of measurable functions on $M$ and $N$ respectively, stable under truncation (see [3, pp. 1037-1038] for details) and $W_{M}$ and $W_{N}$ some semi-norms on $\mathcal{F}_{M}$ and $\mathcal{F}_{N}$ respectively. One can then write $\left(S_{\psi}^{p}\right)$ inequalities, $F$-Sobolev inequalities, etc. on $M$ and $N$ simply replacing $\||\nabla f|\|_{p}$ by $W_{M}(f)$ (resp. $\||\nabla g|\|_{p}$ by $\left.W_{N}(g)\right)$, and $\operatorname{Lip}_{0}(M)\left(\operatorname{resp} . \operatorname{Lip}_{0}(N)\right)$ by $\mathcal{F}_{M}\left(\right.$ resp. $\left.\mathcal{F}_{N}\right)$ in the above definitions.

Consider now the product space $(M \times N, \mu \times \nu)$, and let $\mathcal{F}$ be a class of measurable functions on $M \times N$, such that, if $u \in \mathcal{F}$ then $u(\cdot, y) \in \mathcal{F}_{M}$ for $\nu$-almost every $y \in N$, and $u(x, \cdot) \in \mathcal{F}_{N}$ for $\mu$-almost every $x \in M$. Let $W_{M \times N}$ be a semi-norm on $\mathcal{F}$. We must assume the following three axioms:

(i) There exists $\alpha>0$ such that for all $u \in \mathcal{F}$

$$
W_{M \times N}^{p}(u) \geq \alpha\left(\int_{N} W_{M}^{p}(u(\cdot, y)) d \nu(y)+\int_{M} W_{N}^{p}(u(x, \cdot)) d \mu(x)\right) .
$$

This axiom means that the functional $W_{M \times N}$ is adapted to the product structure.

(ii) Fix $\rho>1$; for $k \in \mathbb{Z}$, and $f \in \mathcal{F}_{M}$, set

$$
f_{\rho, k}:=\min \left\{\left(|f|-\rho^{k}\right)_{+}, \rho^{k}(\rho-1)\right\} .
$$


Then one should have

$$
W_{M}(f)^{p} \geq \beta \sum_{k \in \mathbb{Z}} W_{M}\left(f_{\rho, k}\right)^{p},
$$

for any $f \in \mathcal{F}_{M}$ and some $\beta>0$. The functional $W_{N}$ should satisfy a similar property, possibly for a different $\rho$.

This axiom is called $\left(H_{p}^{\rho}\right)$ in the terminology of [3]. It plays a role in the proof of the equivalence between the $(p, \psi)$ and the $F$-Sobolev inequality in $L^{p}$ when applying a truncation argument (Proposition 2.1).

(iii) This condition is imposed on only one of the functionals (say, $W_{N}$ ) and it reads: if $u \in \mathcal{F}$, then

$$
f(x):=\left(\int_{M}|u(x, \cdot)|^{p} d \mu(x)\right)^{\frac{1}{p}}
$$

belongs to $\mathcal{F}_{N}$ and

$$
\int_{M} W_{N}^{p}(u(x, \cdot)) d \mu(x) \geq \gamma W_{N}^{p}(f)
$$

for some $\gamma>0$.

This axiom is forced upon us by the estimate (3.5) in the proof of Proposition 3.1.

If all axioms $(i),(i i)$ and $(i i i)$ are satisfied, then one can prove along the same lines a generalization of Theorem 1.1.

It has been shown in [3] that axiom (ii) is satisfied for a variety of local and non-local gradients. As for (iii), consider first the case of local gradients, e.g. $W_{N}(f)$ is the $L^{p}$ norm of the "carré du champ" of a diffusion semi-group, or

$$
W_{N}(f)=\left(\int_{N}\left(\sum_{i=1}^{l}\left|X_{i} f\right|^{2}\right)^{\frac{p}{2}} d \nu\right)^{\frac{1}{p}}
$$

where $\left\{X_{i}, i=1, \ldots, l\right\}$ is a family of vector fields on $N$. Then $(i i i)$ follows from the property that for a local gradient $\nabla, f \in \operatorname{Lip}_{0}(M)$, and $\alpha>0$,

$$
\left.\left.|\nabla| f\right|^{\alpha}|\leq \alpha| f\right|^{\alpha-1}|\nabla f| .
$$

Applying then Hölder's inequality, one gets (iii) following the same lines as in the proof of Proposition 3.1. 
In the non-local case, say

$$
W_{N}(f)=\left(\int_{N} \int_{N}\left|f\left(\cdot, y_{1}\right)-f\left(\cdot, y_{2}\right)\right|^{p} K\left(y_{1}, y_{2}\right) d \mu\left(y_{1}\right) d \mu\left(y_{2}\right)\right)^{\frac{1}{p}}
$$

where $K$ is a non-negative kernel such that

$$
\int_{N} K(x, y) d \mu(y)+\int_{N} K(x, y) d \mu(x) \leq C<+\infty,
$$

axiom (iii) is a consequence of the elementary inequality

$$
\begin{gathered}
\left|\left(\int_{M}\left|f\left(x, y_{1}\right)\right|^{p} d \mu(x)\right)^{\frac{1}{p}}-\left(\int_{M}\left|f\left(x, y_{2}\right)\right|^{p} d \mu(x)\right)^{\frac{1}{p}}\right| \\
\leq\left(\int_{M}\left|f\left(x, y_{1}\right)-f\left(x, y_{2}\right)\right|^{p} d \mu(x)\right)^{\frac{1}{p}} .
\end{gathered}
$$

\section{Appendix: Euclidean inequalities.}

Let us first observe that our method yields yet another proof of the Sobolev inequality in $\mathbb{R}^{n}$ (see for instance [13, pp. 162-164] for the classical proof, and [52] for an alternative proof). Start with

$$
2 \sup _{t \in \mathbb{R}}|f(t)| \leq \int_{\mathbb{R}}\left|f^{\prime}(t)\right| d t
$$

which is true for any $f \in \operatorname{Lip}_{0}(\mathbb{R})$ and which obviously implies the onedimensional $L^{1}$ Moser inequality:

$$
2 \int_{\mathbb{R}} f^{2}(t) d t \leq \int_{\mathbb{R}}|f(t)| d t \int_{\mathbb{R}}\left|f^{\prime}(t)\right| d t .
$$

Now, Proposition 3.1, applied $n$ times, yields the $n$-dimensional $L^{1}$ Moser inequality

$$
C_{n} \int_{\mathbb{R}^{n}}|f(x)|^{1+\frac{1}{n}} d x \leq\left(\int_{\mathbb{R}^{n}}|f(x)| d x\right)^{1 / n} \int_{\mathbb{R}^{n}}|\nabla f(x)| d x .
$$

It follows from the results of Section 2 or [3] that (8.1) is equivalent to the regular Sobolev inequality $\left(S_{n}^{1}\right)$ (this can be seen also in a more direct way by going through the isoperimetric inequality (1.1)). Hence, it implies all the $\left(S_{n}^{p}\right), 1 \leq p \leq+\infty$ (see Introduction for the meaning of $\left(S_{n}^{p}\right)$ if $p \geq n$ ). 
The above simple-minded procedure can be applied to obtain also the $n$-dimensional $L^{p}$ Moser inequality and, surprisingly enough, gives for $1 \leq$ $p \leq 2$ a constant that grows with $n$ at a correct rate. Let us start with the one-dimensional $L^{2}$ Moser inequality

$$
A_{1} \int_{\mathbb{R}} f^{6}(t) d t \leq\left(\int_{\mathbb{R}}|f(t)|^{2} d t\right)^{2} \int_{\mathbb{R}}\left|f^{\prime}(t)\right|^{2} d t
$$

with the sharp constant $A_{1}=\frac{\pi^{2}}{4}$ that was computed by Nagy [55]. Assuming that we have already the $n$-dimensional Moser inequality

$$
A_{n} \int_{\mathbb{R}^{n}}|f(x)|^{2+\frac{4}{n}} d x \leq\left(\int_{\mathbb{R}^{n}}|f(x)|^{2} d x\right)^{2 / n} \int_{\mathbb{R}^{n}}|\nabla f(x)|^{2} d x,
$$

with some constant $A_{n}>0$, let us compute $A_{n+1}$. Indeed, (8.2) is equivalent to the $F$-Sobolev inequality with $F(v)=A_{n} v^{2 / n}$. Therefore, Proposition 3.1 applied to $M=\mathbb{R}^{n}$ and $N=\mathbb{R}$ yields the $H$-Sobolev inequality on $\mathbb{R}^{n+1}$ with

$$
H(v)=\inf _{r>0}\left(A_{n} r^{\frac{2}{n}}+A_{1}\left(\frac{v}{r}\right)^{2}\right) .
$$

Evaluating this infimum, which is attained at $r=\left(\frac{A_{1} n v^{2}}{A_{n}}\right)^{\frac{n}{2(n+1)}}$, one obtains

$$
H(v)=A_{n}^{\frac{n}{n+1}}\left(A_{1} n\right)^{\frac{1}{n+1}}\left(1+\frac{1}{n}\right) v^{\frac{2}{n+1}},
$$

that is the Moser inequality holds in $\mathbb{R}^{n+1}$ with the constant

$$
A_{n+1}=A_{n}^{\frac{n}{n+1}}\left(A_{1} n\right)^{\frac{1}{n+1}}\left(1+\frac{1}{n}\right)
$$

One easily obtains by induction that

$$
A_{n}=A_{1} n=\frac{\pi^{2}}{4} n
$$

It is shown in [6] that the best constant $A_{n}^{*}$ in the Moser inequality has the following asymptotic:

$$
A_{n}^{*} \sim \frac{\pi e}{2} n, \quad n \rightarrow \infty .
$$

Hence, our approach gives the correct linear rate of growth of $A_{n}$ as $n \rightarrow \infty$. Note also that $A_{2}=\frac{\pi^{2}}{2}=\pi \cdot 1.5708 \ldots$ whereas $A_{2}^{*}=\pi \cdot 1.8623 \ldots$ (see [67]).

For $p \neq 2$, the above argument does not yield the correct asymptotic order for the best constant of the $n$-dimensional $L^{p}$ Moser inequality, which 
is known to be $n^{\frac{p}{2}}$ (see [6]). In the case $1 \leq p<2$, the origin of the difficulty is the constant $c_{p}$ in the proof of Proposition 3.1. One can overcome this difficulty by defining a modified length of the gradient in the following way:

$$
|\nabla f|_{p}(x):=\left(\sum_{i=1}^{n}\left|\frac{\partial f}{\partial x_{i}}(x)\right|^{p}\right)^{\frac{1}{p}}
$$

(a similar idea was used in [45] in a discrete setting). Using the inequality

$$
\left(\sum_{i=1}^{n} a_{i}^{p}\right)^{1 / p} \leq n^{\frac{1}{p}-\frac{1}{2}}\left(\sum_{i=1}^{n} a_{i}^{2}\right)^{1 / 2}, \quad 1 \leq p<2
$$

one sees immediately

$$
\int_{\mathbb{R}^{n}}|\nabla f|_{p}^{p} d x \leq n^{1-\frac{p}{2}} \int_{\mathbb{R}^{n}}|\nabla f|^{p} d x
$$

On the other hand, if one rewrites Proposition 3.1 with $M=\mathbb{R}^{n}$ endowed with the functional $\int_{\mathbb{R}^{n}}|\nabla f|_{p}^{p} d x$, and $N=\mathbb{R}$, one finds that the constant $c_{p}$ disappears.

Now starting with the one-dimensional $L^{p}$ Moser inequality with constant $A_{1, p}$ and applying $n$ times this version of Proposition 3.1, one obtains

$$
\tilde{A}_{n, p} \int_{\mathbb{R}^{n}}|f(x)|^{p+\frac{p^{2}}{n}} d x \leq\left(\int_{\mathbb{R}^{n}}|f(x)|^{p} d x\right)^{p / n} \int_{\mathbb{R}^{n}}|\nabla f|_{p}^{p}(x) d x
$$

with

$$
\tilde{A}_{n, p}=n \tilde{A}_{1, p}=n A_{1, p} .
$$

Taking (8.3) into account yields

$$
A_{n, p} \int_{\mathbb{R}^{n}}|f(x)|^{p+\frac{p^{2}}{n}} d x \leq\left(\int_{\mathbb{R}^{n}}|f(x)|^{p} d x\right)^{p / n} \int_{\mathbb{R}^{n}}|\nabla f|^{p}(x) d x
$$

with

$$
A_{n, p}=\tilde{A}_{n, p} n^{\frac{p}{2}-1}=\tilde{A}_{1, p} n n^{\frac{p}{2}-1}=A_{1, p} n^{\frac{p}{2}} .
$$

which gives the correct asymptotic order in $n$.

In the case $p>2$, the constant $c_{p}$ is 1 , but the above computation leads us only to $A_{n, p}=n A_{1, p} \asymp n$ while the correct order for the best constant is $n^{\frac{p}{2}}$. Note added in proof: in a preprint called Estimates on Moser embedding, William Beckner has recently been able to treat also this case.

Acknowledgement: The first-named author thanks Michel Ledoux for useful comments and references, Emmanuel Hebey for providing reference [6], William Beckner for commenting it, Yvan Martel and Frank Merle for providing reference [67]. 


\section{References.}

[1] Bakry D., L'hypercontractivité et son utilisation en théorie des semigroupes, in: "Lectures on probability theory. Lectures from the Twentysecond Saint-Flour Summer School held July 9-25, 1992", ed. P. Bernard, Lecture Notes in Mathematics 1581, Springer-Verlag, Berlin, 1994.

[2] Bakry D., Concordet D., Ledoux M., Optimal heat kernel bounds under logarithmic Sobolev inequalities, ESAIM Probability and Statistics, 1 (1997) 391-407.

[3] Bakry D., Coulhon T., Ledoux M., Saloff-Coste L., Sobolev inequalities in disguise, Indiana Univ. Math. J., 124 (1995) no.4, 1033-1074.

[4] Barlow M.T., Coulhon T., Grigor'yan A., Manifolds and graphs with slow heat kernel decay, Invent. Math., 144 (2001) 609-649.

[5] Beckner W., Geometric asymptotics and the logarithmic Sobolev inequality, Forum Math., 11 (1999) 105-137.

[6] Beckner W., Asymptotic estimates for Gagliardo-Nirenberg embedding constants, Potential Anal., 17 (2002) no.3, 253-266.

[7] Bendikov A., Symmetric stable semigroups on the infinite dimensional torus, Expo. Math., 13 (1995) 39-80.

[8] Bendikov A., Saloff-Coste L., On- and off-diagonal heat kernel behaviors on certain infinite dimensional local Dirichlet spaces, Amer. J. Math., 122 (2000) 1205-1263.

[9] Benedek A., Panzone R., The spaces $L_{p}$ with mixed norms, Duke Math. J., 28 (1961) 301-324.

[10] Biroli M., Maheux P., Inégalités de Sobolev logarithmiques et de type Nash pour des semi-groupes sous-markoviens symétriques, preprint.

[11] Bobkov S.G., An isoperimetric inequality on the discrete cube, and an elementary proof of the isoperimetric inequality in Gauss space, Ann. Probab., 25 (1997) no.1, 206-214.

[12] Bobkov S.G., Houdré C., Some connections between isoperimetric and Sobolev-type inequalities, Mem. Amer. Math. Soc., 129 (1997) no. 616, viii+111pp. 
[13] Brezis H., "Analyse fonctionnelle, Théorie et applications", Masson, 1983.

[14] Carron G., Inégalités isopérimétriques sur les variétés riemanniennes, Thesis, University of Grenoble, 1994.

[15] Carron G., Inégalités isopérimétriques de Faber-Krahn et conséquences, in: "Actes de la table ronde de géométrie différentielle (Luminy, 1992)", Collection SMF Séminaires et Congrès 1, 1996. 205-232.

[16] Chavel I., "Eigenvalues in Riemannian geometry", Academic Press, New York, 1984.

[17] Chavel I., "Isoperimetric inequalities: differential geometric and analytic perspectives", Cambridge Tracts in Mathematics 145, Cambridge University Press, 2001.

[18] Chavel I., Feldman E.A., Modified isoperimetric constants, and large time heat diffusion in Riemannian manifolds, Duke Math. J., 64 (1991) no.3, 473-499.

[19] Chung F.R.K., Grigor'yan A., Yau S.-T., Higher eigenvalues and isoperimetric inequalities on Riemannian manifolds and graphs, Comm. Anal. Geom, 8 (2000) no.5, 969-1026.

[20] Concordet D., Inégalités de Sobolev et hypercontractivité, unpublished manuscript, 1994.

[21] Coulhon T., Dimension à l'infini d'un semi-groupe analytique, Bull. Sc. Math., 114 (1990) no.3, 485-500.

[22] Coulhon T., Noyau de la chaleur et discrétisation d'une variété riemannienne, Israël J. Math., 80 (1992) 289-300.

[23] Coulhon T., Dimensions at infinity for Riemannian manifolds, Potential Anal., 4 (1995) no.5, 335-344.

[24] Coulhon T., Espaces de Lipschitz et inégalités de Poincaré, J. Funct. Anal., 136 (1996) no.1, 81-113.

[25] Coulhon T., Ultracontractivity and Nash type inequalities, J. Funct. Anal., 141 (1996) no.2, 510-539. 
[26] Coulhon T., Large time behaviour of heat kernels on Riemannian manifolds: fast and slow decays, in: "Journées équations aux dérivées partielles, St-Jean-de-Monts", (1998) II,1-II,12.

[27] Coulhon T., Grigor'yan A., Pittet Ch., A geometric approach to ondiagonal heat kernel lower bounds on groups, Ann. Inst. Fourier, Grenoble, 51 (2001) no.6, 1763-1827.

[28] Coulhon T., Holopainen I., Saloff-Coste L., Harnack inequality and hyperbolicity for subelliptic $p$-Laplacians with applications to Picard type theorems, Geom. and Funct. Anal., 11 (2001)

[29] Coulhon T., Ledoux M., Isopérimétrie, décroissance du noyau de la chaleur et transformations de Riesz: un contre-exemple, Ark. Mat., 32 (1994) 63-77.

[30] Coulhon T., Saloff-Coste L., Isopérimétrie pour les groupes et les variétés, Revista Matemática Iberoamericana, 9 (1993) no.2, 293314.

[31] Coulhon T., Saloff-Coste L., Variétés riemanniennes isométriques à l'infini, Revista Matemática Iberoamericana, 11 (1995) no.3, 687726 .

[32] Davies E.B., Explicit constants for Gaussian upper bounds on heat kernels, Amer. J. Math., 109 (1987) 319-334.

[33] Davies E.B., Gaussian upper bounds for the heat kernel of some secondorder operators on Riemannian manifolds, J. Funct. Anal., 80 (1988) 16-32.

[34] Davies E.B., "Heat kernels and spectral theory", Cambridge University Press, 1989.

[35] Davies E.B., Simon B., Ultracontractivity and the heat kernel for Schrödinger operators and Dirichlet Laplacians, J. Funct. Anal., 59 (1984) 335-395.

[36] Federer H., "Geometric measure theory", Berlin: Springer, 1969.

[37] Grigor'yan A., Isoperimetric inequalities for Riemannian products, (in Russian) Mat. Zametki, 38 (1985) no.4, 617-626. Engl. transl. Math. Notes, 38 (1985) 849-854. 
[38] Grigor'yan A., On the existence of positive fundamental solution of the Laplace equation on Riemannian manifolds, (in Russian) Matem. Sbornik, 128 (1985) no.3, 354-363. Engl. transl. Math. USSR Sb., 56 (1987) 349-358.

[39] Grigor'yan A., The heat equation on non-compact Riemannian manifolds, (in Russian) Matem. Sbornik, 182 (1991) no.1, 55-87. Engl. transl. Math. USSR Sb., 72 (1992) no.1, 47-77.

[40] Grigor'yan A., Heat kernel upper bounds on a complete non-compact manifold, Revista Matemática Iberoamericana, 10 (1994) no.2, 395452 .

[41] Grigor'yan A., Isoperimetric inequalities and capacities on Riemannian manifolds, Operator Theory: Advances and Applications, 109 (1999) 139-153.

[42] Grigor'yan A., Estimates of heat kernels on Riemannian manifolds, in: "Spectral Theory and Geometry. ICMS Instructional Conference, Edinburgh 1998", ed. B.Davies and Yu.Safarov, London Math. Soc. Lecture Note Series 273, Cambridge Univ. Press, 1999. 140-225.

[43] Grigor'yan A., Analytic and geometric background of recurrence and non-explosion of the Brownian motion on Riemannian manifolds, Bull. Amer. Math. Soc., 36 (1999) 135-249.

[44] Hebey E., "Sobolev spaces on Riemannian manifolds", Lecture Notes Math. 1635, Springer, 1996.

[45] Houdré C., Tetali P., Concentration of measures for products of Markov kernels and graph products via functional inequalities, Combin. Probab. Comput., 10 (2001) no.1, 1-28.

[46] Kavian O., Kerkyacharian G., Roynette B., Quelques remarques sur l'ultracontractivité, J. Funct. Anal., 111 (1993) no.1, 155-196.

[47] Ledoux M., The geometry of Markov diffusion generators, Ann. Fac. Sci. Toulouse Math. (6), 9 (2000) no.2, 305-366.

[48] Levin D., Solomyak M., The Rozenblum-Lieb-Cwikel inequality for Markov generators, J. d'Analyse Math., 71 (1997) 173-193.

[49] Li P., On the Sobolev constant and the $p$-spectrum of a compact Riemannian manifold, Ann. Sci. Ecole Norm. Sup. Paris, 13 (1980) 419-435. 
[50] Li P., Curvature and function theory on Riemannian manifolds, Surveys in Diff. Geom., VII (2000) 375-432.

[51] Li P., Schoen R., Yau S.-T., On the isoperimetric inequality for minimal surfaces, Ann. Scuola Norm. Sup. Pisa, 11 (1984) 237-244.

[52] Malý J., Pick L., An elementary proof of sharp Sobolev embeddings, Proc. Amer. Math. Soc., 130 (2002) no.2, 555-563.

[53] Maz'ya V.G., "Sobolev spaces", (in Russian) Izdat. Leningrad Gos. Univ. Leningrad, 1985. Engl. transl. Springer, 1985.

[54] Maz'ya V.G., Classes of domains, measures and capacities in the theory of differentiable functions, in: "Analysis, III", Encyclopaedia Math. Sci. 26, Springer, Berlin, 1991. 141-211.

[55] Nagy B.Sz., Über integralungleichungen zwischen einer funktion und ihrer ableitung, Acta Sci. Math. (Szeged), 10 (1941) 64-74.

[56] Pittet Ch., Saloff-Coste L., Amenable groups, isoperimetric profiles and random walks, in: "Geometric group theory down under. Proceedings of a special year in geometric group theory, Canberra, Australia, 1996", ed. J. Cossey, C.F. Miller III, W.D. Neumann and M. Shapiro, Walter De Gruyter, 1999.

[57] Röckner M., Wang F.Y., Ultracontractivity and supercontractivity of Markov semigroups, preprint.

[58] Schoen R., Yau S.-T., "Lectures on Differential Geometry", Conference Proceedings and Lecture Notes in Geometry and Topology 1, International Press, 1994.

[59] Tillich J.-P., Edge isoperimetric inequalities for product graphs, in: "Selected topics in discrete mathematics (Warsaw, 1996)", Discrete Math., 213 (2000) no.1-3, 291-320.

[60] Tomisaki M., Comparison theorems on Dirichlet norms and their applications, Forum Math., 2 (1990) 277-295.

[01] Varopoulos N.Th., Hardy-Littlewood theory for semigroups, J. Funct. Anal., 63 (1985) no.2, 240-260.

[62] Varopoulos N.Th., Isoperimetric inequalities and Markov chains, $J$. Funct. Anal., 63 (1985) no.2, 215-239. 
[63] Varopoulos N.Th., Analysis on nilpotent groups, J. Funct. Anal., 66 (1986) 406-431.

[64] Varopoulos N.Th., Groups of superpolynomial growth, in: "Proceedings of the ICM satellite conference on Harmonic analysis", Springer, 1991.

[65] Varopoulos N.Th., Saloff-Coste L., Coulhon T., "Analysis and geometry on groups", Cambridge University Press, Cambridge, 1992.

[66] Wang F.-Y., Functional inequalities for empty essential spectrum, $J$. Funct. Anal., 170 (2000) no.1, 219-245.

[67] Weinstein M.I., Nonlinear Schrödinger equations and sharp interpolation estimates, Comm. Math. Phys., 87 (1983) 567-576.

[68] Yau S.-T., Isoperimetric constant and the first eigenvalue of a compact Riemannian manifold, Ann. Sci. Ecole Norm. Sup., 4th serie, 8 (1975) no.4, $487-507$.

[69] Yau S.-T., Survey on partial differential equations in differential geometry, Ann. Math. Studies, 102 (1982) 3-70.

[70] Zegarlinski B., All that is Sobolev inequality, preprint.

Université de Cergy-Pontoise

95000 CERGY

FRANCE

E-mail address: thierry.coulhon@math.u-cergy.fr

IMPERIAL COLLEGE

LONDON SW7 2BZ

ENGLAND

E-mail address: a.grigoryan@ic.ac.uk

Université de Cergy-Pontoise

95000 CERGY

FRANCE

E-mail address: daniel.levin@math.u-cergy.fr

RECEIVEd JANuARY 4, 2002. 